\title{
Merqury: reference-free quality, completeness, and phasing assessment for genome assemblies
}

\author{
Arang Rhie* ${ }^{*}$, Brian P. Walenz, Sergey Koren and Adam M. Phillippy
}

\author{
* Correspondence: arang.rhie@nih. \\ gov \\ Genome Informatics Section, \\ Computational and Statistical \\ Genomics Branch, National Human \\ Genome Research Institute, National \\ Institutes of Health, Bethesda, MD, \\ USA
}

\begin{abstract}
Recent long-read assemblies often exceed the quality and completeness of available reference genomes, making validation challenging. Here we present Merqury, a novel tool for reference-free assembly evaluation based on efficient k-mer set operations. By comparing k-mers in a de novo assembly to those found in unassembled high-accuracy reads, Merqury estimates base-level accuracy and completeness. For trios, Merqury can also evaluate haplotype-specific accuracy, completeness, phase block continuity, and switch errors. Multiple visualizations, such as k-mer spectrum plots, can be generated for evaluation. We demonstrate on both human and plant genomes that Merqury is a fast and robust method for assembly validation.

Keywords: Genome assembly, Assembly validation, Benchmarking, K-mers, Haplotype phasing, Trio binning
\end{abstract}

\section{Background}

With recent advances in long-read [1-3] and long-range sequencing technologies [4-6], new assembly pipelines are generating more continuous, complete, and accurate diploid genome assemblies than ever before [4, 7-14].

However, de novo assembled genomes are difficult to validate due to the lack of a known truth. Existing methods use Illumina reads to infer base-level accuracy by aligning the reads to the assembly for evaluation [15]. Base errors in the consensus are detected as variants (SNPs or small indels) when aligning the short reads. However, this method is heavily reliant on the short-read mapping, which could be biased in repetitive regions, under-collapsed regions, or regions of low consensus accuracy. For measuring completeness and false duplications, near-universal single-copy orthologs (BUSCOs) [16] have been widely used to evaluate the gene content of assemblies. BUSCO is robust for species that have been widely studied, such as human and mouse. However, this analysis can be inaccurate when the newly assembled genome contains true copy number or sequence variants that were not considered when building the

(c) The Author(s). 2020 Open Access This article is licensed under a Creative Commons Attribution 4.0 International License, which permits use, sharing, adaptation, distribution and reproduction in any medium or format, as long as you give appropriate credit to the original author(s) and the source, provide a link to the Creative Commons licence, and indicate if changes were made. The images or other third party material in this article are included in the article's Creative Commons licence, unless indicated otherwise in a credit line to the material. If material is not included in the article's Creative Commons licence and your intended use is not permitted by statutory regulation or exceeds the permitted use, you will need to obtain permission directly from the copyright holder. To view a copy of this licence, visit http://creativecommons.org/licenses/by/4.0/. The Creative Commons Public Domain Dedication waiver (http://creativecommons.org/publicdomain/zero/1.0/) applies to the data made available in this article, unless otherwise stated in a credit line to the data. 
initial BUSCO gene set. In addition, BUSCO only examines conserved single-copy genes and fails to evaluate the most difficult-to-assemble regions of the genome.

In contrast, k-mers (genomic substrings of length $k$ ) can be used in a reference-free manner for assessing genome assembly quality metrics. Genome assembly validation via k-mer copy number analysis was introduced by Mapleson et al. in their KAT tool [17], which enables visual inspection of k-mer spectra plots to identify artificial duplications and missing sequences. Merqury takes much of its inspiration from the ideas introduced by KAT. QUAST-LG [18] is another assembly validation tool that provides both BUSCO and reference-based k-mer measurements, as well as alignment metrics against a closely related reference genome. However, many of QUAST's metrics are based on the reference alignment, which incorrectly reports true variants in the assembled genome as potential mis-assemblies. A recent extension to the QUAST toolkit combines evidence from both k-mers and long reads to better identify certain classes of structural mis-assembly [19].

Assessing haplotype phasing without a truth set is also challenging. Diploid genome assemblers generate both primary and alternate assemblies representing the two haplotypes. The primary assembly is typically a pseudo-haplotype that captures both the homozygous regions along with a single copy of the heterozygous alleles. Such a pseudo-haplotype does not guarantee long-range phasing, so to estimate phase block statistics, the alternate alleles must be mapped back to the primary assembly to determine regions corresponding to the primary-alternate haplotype phase blocks [9]. However, this can be challenging when the alternate alleles do not map well to the primary sequence due to high sequence divergence or mis-assemblies. Moreover, long-read assemblies often collapse regions of low heterozygosity, which are excluded when calculating phase block statistics, thus over-representing the correctness.

Alternative methods report phasing statistics from small variants (mostly SNPs) called with short-read mapping [8,20-23], or use benchmark genomes that have curated, phased variation call sets [24-27]. Both methods rely on a reference sequence as the primary source to detect heterozygous variations. However, these reference sequences are incomplete, and recent studies have demonstrated the shortcomings of the current human reference genome and variant call sets [8, 21, 28]. For example, the highly variable major histocompatibility complex (MHC) is excluded from the Genome in a Bottle (GIAB) and Global Alliance for Genomic Health (GA4GH) reference panels [26] due to its repetitive nature and need of a specialized mapping strategy to account for the high allelic diversity [29]. Moreover, reference-guided strategies require significant manual curation and effort and will not scale as large cohort sequencing projects become common [30,31]. This validation strategy is also not applicable to any species without a curated and complete reference [32].

To overcome these limitations, we developed Merqury, which generates assembly assessment metrics using k-mers alone. Merqury compares a set of k-mers derived from unassembled, high-accuracy sequencing reads to a genome assembly for evaluation. Merqury builds upon the k-mer-based analyses introduced by KAT but adds new functionality for evaluating the accuracy of phased diploid genome assemblies (Table 1). Unique to Merqury, the generated metrics include consensus quality (QV) and k-mer completeness, and when parental genomic sequences are available (either assembled or unassembled), Merqury can output haplotype completeness, phase block statistics, 
Table 1 Feature comparison of KAT and Merqury

\begin{tabular}{lll}
\hline & KAT & Merqury \\
\hline K-mer analysis & & $Y$ \\
Count & $Y$ & $Y$ \\
Filter & $Y$ & $Y$ \\
Compare & $Y$ & - \\
\%GC & $Y$ & - \\
Fitting distributions & - & $Y$ \\
Other set operations, querying & & \\
Assembly statistics & $Y$ & - \\
Finding repetitive regions & $Y$ & - \\
Contamination detection & $Y$ & $Y$ \\
K-mer copy-number spectrum & - & $Y$ \\
K-mer assembly, haplotype spectrum & - & $Y$ \\
Consensus quality (QV) & - & $Y$ \\
K-mer completeness & - & $Y$ \\
Phase blocks and switch error rate & & $Y$ \\
Plotting & - & $Y$ \\
Stacked histograms & $Y$ & $Y$ \\
Unstacked histograms & - & $Y$ \\
K-mer coverage & $Y$ & $Y$ \\
\%GC Heatmaps & - & $Y$ \\
Hap-mer fractions (blob plots) & $Y$ & $Y$ \\
Nrowser tracks (BED, TDF) & $Y$ NGx contig/block sizes & $Y$ \\
\hline
\end{tabular}

switch error rates, and visual representations of phase consistency for the child's genome. This includes TDF (or BED) features that can be displayed in a genome browser for visualizing the presence of k-mer classes across a genome (e.g., the k-mers inherited from a parental genome). We show that Merqury's k-mer-based assembly validation produces comparable or better results than existing methods, such as BUSCO gene completeness and mapping-based measurements.

\section{Results}

To demonstrate the ability of Merqury to evaluate the accuracy, completeness, and phasing of an assembly, we first applied it to an Arabidopsis thaliana F1 hybrid [9], for which the parental strains (Col-0 and Cvi-0, simplified as $\mathrm{Col}$ and Cvi) have also been sequenced. For a comparison of multiple assemblies, we demonstrate Merqury on haplotype-resolved (TrioCanu [10]), pseudo-haplotype (FALCON-Unzip [9]), and mixed-haplotype (Canu [33]) assemblies of this hybrid genome. Total assembly size is typically used as a rough measure of haplotype completeness. For example, the TrioCanu haplotype assemblies have similar total bases, 122 124 Mbp (Table 2), close to the expected haploid genome size of $130 \mathrm{Mbp}$, indicating the haplotype assemblies are well balanced (assuming haplotype-specific bases are evenly inherited). In comparison, the primary assembly of FALCON-Unzip has $\sim 35 \mathrm{Mbp}$ bases more than the alternate assembly. However, it is difficult to understand where this difference originates 


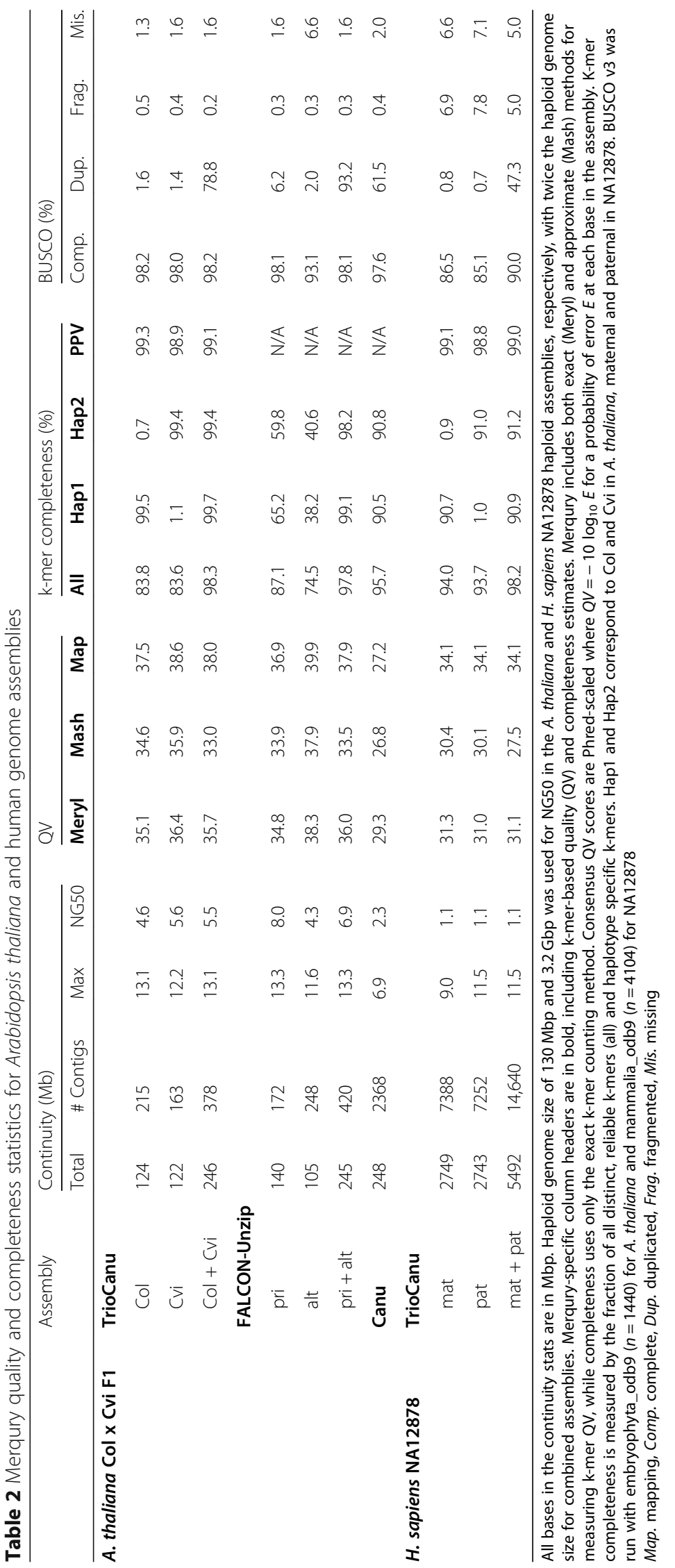


from the assembly size alone. The mixed-haplotype Canu assembly, in comparison, is $\sim 100 \mathrm{Mbp}$ larger than the expected genome size of $130 \mathrm{Mb}$. Again, we can assume this assembly resolved both haplotypes, but since the haplotypes have been combined in a single assembly, we cannot know the composition from the size and continuity measures alone. In the following sections, we describe how Merqury's statistics and plots can be used to dissect and understand these assemblies.

\section{Copy number spectrum}

We counted k-mers from Illumina whole-genome sequencing of the A. thaliana F1 hybrid, as well as from each assembly, using Meryl, a k-mer counting tool we extended to support k-mer set operations for Merqury (Methods). The copy number spectrum plot, known as "spectra-cn" plot [17] tracks the multiplicity of each k-mer found in the Illumina read set (Fig. 1a) and colors it by the number of times it is found in a given assembly (Fig. 1b). The result is a set of histograms relating k-mer counts in the read set to their associated counts in the assembly. Here the Illumina dataset (which we will refer to as the "read set") was sequenced to an average coverage of $45 \times$, so we expect a histogram peak near $x=45$ corresponding to k-mers present in both haplotypes, and a peak at half coverage $(x=22)$ representing k-mers found on only one haplotype (Fig. 1a, in practice, these peaks are shifted slightly lower due to the effects of sequencing error and sampling only $l-k+1 k$-mers for each $l$-sized read). We refer to these as 2 -copy and 1-copy k-mers, respectively, to indicate the number of times they appear in the true genome. Additional peaks may appear for polyploid genomes, but for the remainder of this paper, we will assume a diploid genome.

Thus, when a k-mer is found approximately 22 times in the A. thaliana read set, we expect it to be found only once in the assembly, as it is likely a 1-copy, haplotypespecific (heterozygous) sequence (Fig. 1b). In the spectra-cn plot, k-mers are colored based on their count in the assembly. For a complete haplotype-resolved assembly, where even the homozygous part of the genome is included in both haplotypes, we expect most k-mers in the 2-copy peak to be found twice in the assembly (Fig. 1b). For partially phased assemblies, 2-copy k-mers may be found either once or twice in the assembly (e.g., Fig. 2), depending on which homozygous sequences of the genome were separated and which were collapsed. In contrast, a pseudo-haplotype collapses homozygous alleles, so 2-copy k-mers are expected to appear only once in the assembly. One notable exception is haplotype-specific duplications, which can occur in two copies on the same haplotype, and thus may also appear in two copies in a pseudo-haplotype assembly.

Assuming no serious biases in the read set, a clean spectra-cn plot is a necessary, but not sufficient, condition of assembly quality. Quoting KAT author Bernardo Clavijo "if your spectra look right, your assemblies could still be wrong, but if your spectra look wrong your assemblies can't be right." That is to say, certain structural errors may not be reflected by the spectra, but any conflict with the copy numbers of the read set indicate assembly problems. The k-mers found only in the read set (black) at low frequency are almost always indicative of sequencing error in the read set; however, higherfrequency k-mers found only in the read set indicate missing sequences in the assembly (e.g., black k-mers within the 1- or 2-copy peaks). Likewise, any k-mers with a higher 


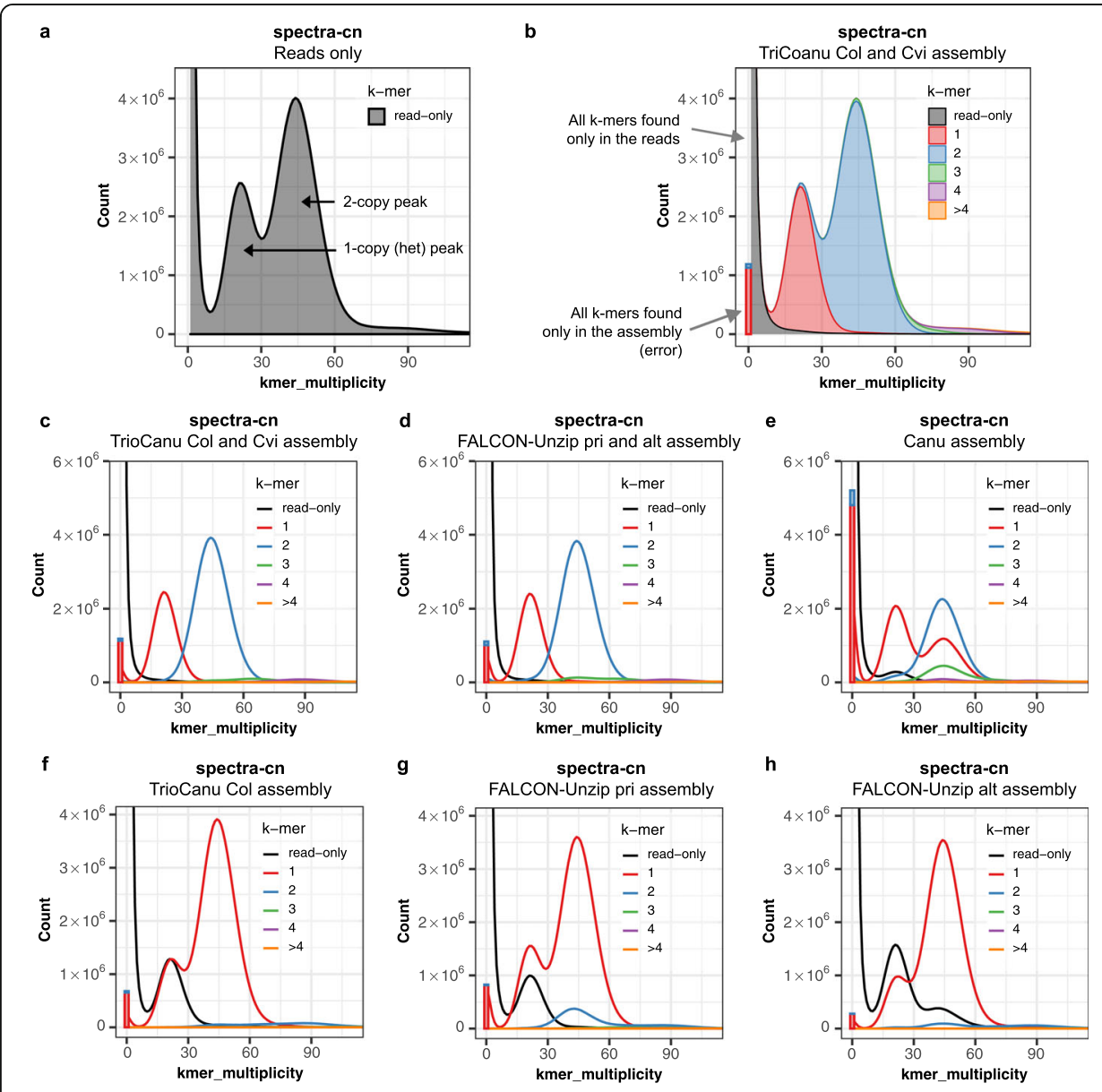

Fig. 1 Merqury copy number spectrum plots for haploid and diploid assemblies of an Arabidopsis thaliana F1 hybrid genome. a Histogram of k-mer multiplicity collected from Illumina reads. By default, Merqury connects the midpoint of each histogram bin with a line, giving the illusion of a smooth curve. The first peak represents 1-copy (heterozygous) k-mers in the genome, and the second peak represents 2-copy kmers originating from homozygous sequence or haplotype-specific duplications. Depth of sequencing coverage determines where these peaks appear. In this example, sequencing coverage is approximately $45 \times$, corresponding to the 2-copy peak. b Copy number spectrum (spectra-cn) of the same k-mers in a plotted as stacked histograms colored by the copy numbers found in the combined TrioCanu assembly. The assembly k-mers absent from the read set (likely to be base errors in the assembly) are plotted as a bar at zero multiplicity, colored by the copy numbers found in the assembly. c Unstacked histograms of $\mathbf{b}$ for visualizing the distribution of k-mer counts per copy numbers found in the assembly. This plot shows an ideal pseudo haplotype assembly. $\mathbf{d}$ Spectra-cn plot of the combined FALCON-Unzip assembly. e Spectracn plot of the mixed-haplotype Canu assembly. Missing single copy k-mers (black) and k-mers from artificial duplications (green, purple, yellow in 30-60x) are noticeable. Note this assembly was not polished and so has a larger error k-mer bar. f Spectra-cn plot of the TrioCanu Col haplotype assembly. Half the single copy $\mathrm{k}$-mers are missing and found in the other haplotype (black). Two-copy k-mers are found once (red) in each haplotype assembly. $\mathbf{g}$ Spectra-cn plot of the FALCON-Unzip primary assembly. $\mathbf{h}$ Spectra-cn plot of the FALCON-Unzip alternate assembly

copy number in the assembly than would be predicted by the read set are indicative of artificial duplications in the assembly, e.g., see the 2-copy k-mers appearing three times in the Canu assembly shown in Fig. 1e.

The bar at the origin of the plots represents k-mers found only in the assembly. From these k-mers, we can estimate an assembly consensus quality value (QV), which represents a log-scaled probability of error for the consensus base calls (Methods). Higher 

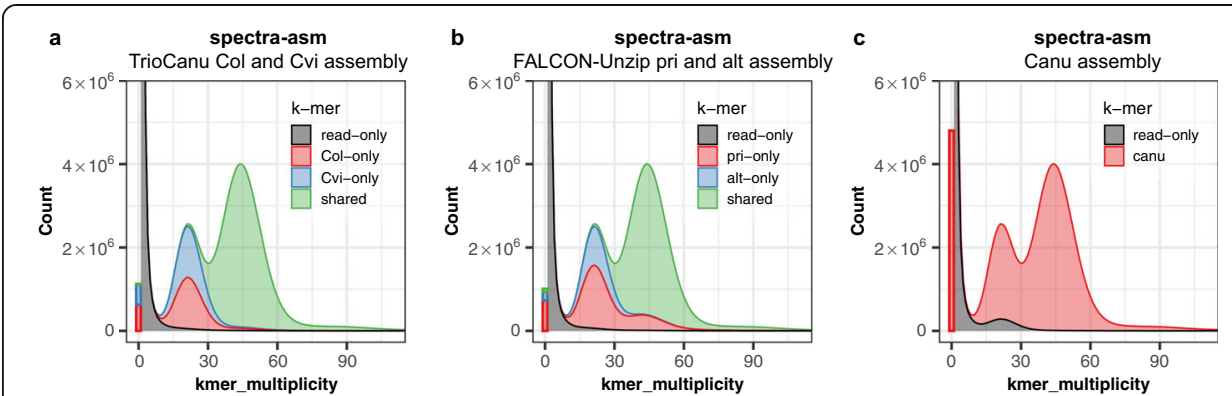

Fig. 2 Merqury assembly spectrum plots for evaluating k-mer completeness. K-mers are colored by their presence in the reads and primary/alternate assemblies. a Distinct k-mer assembly spectrum (spectra-asm) plot of both TrioCanu Col and Cvi haplotype assemblies. This plot shows the assembly-specific (red and blue) and shared portion of k-mers (green). $\mathbf{b}$ Spectra-asm plot of the FALCON-Unzip assembly. The primary assembly has more k-mers (red) compared to the alternate assembly (blue). c Spectra-asm plot of the Canu assembly which is a mixture of both haplotypes. A small fraction of 1-copy k-mers is found only in the reads (black peak around 12 30X), which represents heterozygous variants missing from the assembly

QVs indicate a more accurate consensus, where Q30 corresponds to $99.9 \%$ accuracy, Q40 to 99.99\%, etc. The trio-binned assembly has QV scores of 35 36 for each haploid assembly and 35.7 for the combined version. The FALCON-Unzip assembly has a similar QV score of 35 38 for each haplotype and 36 for the combined version. The error k-mer bar for Canu is much higher than the other two diploid assemblies, as we omitted signal-level polishing [34] from this assembly to show an intermediate assembly product. The estimated QV for this assembly is 29.3 (Table 2).

To better visualize the k-mer distribution by each copy number found in an assembly, we also provide unstacked versions of the spectra-cn histograms (Fig. 1c-e). The 1copy (heterozygous) k-mers appear once in the combined TrioCanu assemblies (red), and 2-copy k-mers twice (blue) as expected (Fig. 1c). The partially phased FALCONUnzip assembly shows a similar distribution to TrioCanu (Fig. 1d), indicative of good k-mer completeness. The lower fraction of 2-copy k-mers found three times in the assembly (green hump under the 2-copy peak) indicates fewer false duplications in the TrioCanu compared to FALCON-Unzip and Canu.

When generating the same spectrum on the mixed-haplotype Canu assembly (Fig. 1e), we can see the assembly has only partially assembled both haplotypes and there is a higher fraction of k-mers missing from the assembly (black). In addition, the assembly has artificial duplications inflating the assembly size. The plot also shows fewer 2-copy k-mers (blue peak) compared to the other assemblies, and a significant fraction of 2copy k-mers appearing only once in the assembly (second red peak), suggesting the mixed-haplotype assembly has partially collapsed the haplotypes. Based on the number of 2-copy k-mers found twice in the assembly, we estimate $42.7 \mathrm{Mbp}$ of homozygous sequence remains un-collapsed, typically at the boundary of heterozygous and homozygous alleles. This partial separation is also evident in the higher number of k-mers appearing three (green), four (purple), and more (yellow) times in the assembly, representing 10.8 million artificially duplicated k-mers that would need to be identified and removed to form a fully collapsed, haploid assembly. This example highlights the benefits of the TrioCanu and FALCON-Unzip approaches for this heterozygous genome. Additional processing with a tool such as purge_dups [35] would be required to convert Canu's mixed haplotypes into a pseudo-haplotype. 
The spectra-cn plots can also be useful for evaluating haploid assemblies, e.g., of a single haplotype from a diploid genome. When plotting the same k-mer spectrum on one haplotype ("Col" in this case), we can see both 1-copy and 2-copy k-mers are now observed just once in the assembly (Fig. 1f, red histogram). This is because the read set represents the full diploid genome, while the assembly isolates a single haplotype. The usual 1-copy peak is now exactly half the size and perfectly overlaps with a peak of missing k-mers (black) that belong to the other haplotype ("Cvi"). In comparison, the pseudo-haplotype FALCON-Unzip primary and alternate assemblies (Fig. 1g. h) show imbalanced peaks, with more assembly k-mers appearing in both the 1- and 2-copy peaks than expected. This suggests that the FALCON-Unzip is erroneously including sequences from both haplotypes into the primary pseudo-haplotype. A similar portion of 1-copy k-mers are missing from the alternate assembly (imbalanced red and black peaks), suggesting that the alternate haplotype is missing some heterozygous variants.

\section{Assembly spectrum}

In the above FALCON-Unzip pseudo-haplotype example (Fig. 1g, h), it is possible to infer that the missing sequences in the alternate assembly are likely found in the primary assembly. However, if there are shared sequences between the two assemblies, it is difficult to know the exact sequence composition. To better address this question, we introduce a new method to show the shared and specific k-mers in each assembly (spectra-asm), instead of showing the overall copy-numbers (Fig. 2). This plot is helpful for measuring diploid assembly completeness as it shows the fraction of $\mathrm{k}$-mers specific to both the primary and alternate assemblies. For example, a perfectly assembled diploid genome is expected to have a balance of k-mers specific to each haplotype representing the heterozygous alleles (exceptions to this include sex chromosomes of different sizes). The spectra-asm of the TrioCanu combined assembly (Fig. 2a) shows such an example, where 1-copy k-mers are specific to each haplotype assembly (red and blue), and the 2-copy k-mers are shared by both assemblies (green). In comparison, the FALCON-Unzip assembly is imbalanced, with more 1-copy and 2-copy k-mers in the primary assembly than expected (Fig. 2b). This imbalance is also evident from Merqury's k-mer completeness metrics for the primary and alternate assemblies (Table 2, completeness "all"). By stacking the histograms, we can confirm the primary assembly contains all the missing k-mers from Fig. $1 \mathrm{~h}$ in the 2-copy peak. Compared to TrioCanu and FALCON-Unzip, Canu does not partition its output into primary and alternates, and so k-mers from both haplotypes are present in the combined assembly. However, the spectra-asm plot shows a few 1-copy k-mers are missing from the assembly (Fig. 2c). This is in concordance with the lower k-mer completeness score in the Canu assembly (95.7\%) compared to the other diploid assemblies (98.3 and 97.8\%, Table 2) and indicates that some heterozygous variants are missing, perhaps as a result of the low-quality, unpolished consensus.

\section{Haplotype-specific k-mers (hap-mers) from trios}

We define a "hap-mer" as a haplotype-specific k-mer that appears exclusively, one or more times, on a single haplotype of the genome. When parental genomes are available, we can use inheritance to estimate a set of hap-mers for the child and evaluate 
haplotype completeness of the assembly. Merqury identifies hap-mers as the set of inherited, parental-specific k-mers (Fig. 3a). Using the parental-specific markers alone may be sufficient for many cases; however, we have found it useful to specifically consider the inherited markers, as only half of the parental specific k-mers are inherited and the non-inherited markers may match an erroneous k-mer in the assembly by chance. Note, this is still only an estimate of the true set of hap-mers in the child's genome, which can also be affected by de novo variants or heterozygous variants that were differentially inherited from the parents. We have implemented efficient k-mer set operations (union, intersection, subtraction, etc.) within Meryl for computing hap-mers and other useful kmer sets (Fig. 3b, Methods). For the A. thaliana F1 hybrid genome, we identified hapmers directly from the genomes of the parental strains. K-mers were grouped based on their presence in the F1 reads alone, the maternal haplotype, the paternal haplotype, or both (Fig. 3c). Because of the high heterozygosity of the F1 (estimated at $0.99 \%$ by GenomeScope [36]), many of the F1's k-mers are hap-mers. A human genome, in comparison, has relatively fewer hap-mers compared to all k-mers in the 1- and 2-copy peaks, with most of the k-mers shared between both haplotypes (Fig. 3d).

\section{Evaluating phasing completeness with hap-mers}

Hap-mers are used to determine phase blocks in Merqury, where a block is defined to be a consistent set of markers originating from the same haplotype. To account for minor base-level errors in the assembly, we do allow some short-range switches to occur within a block, so long as the phase switches back shortly thereafter (Fig. 4a). A benefit of this k-mer approach is that Merqury does not need to rely on the phase blocks as identified by the assembler or a reference variant callset and can quickly compute the blocks on each assembly directly using only the observed haplotype markers. Applying this method to the TrioCanu assembly reported NG50 phase block sizes of 3.6 $\mathrm{Mbp}$ and $5.5 \mathrm{Mbp}$ with $0.3 \%$ per-block switch rate when allowing at most 100 consecutive switches within $20 \mathrm{kbp}$ (Fig. 4b). The FALCON-Unzip assembly has slightly shorter phase block sizes of $3.1 \mathrm{Mbp}$ and $2.5 \mathrm{Mbp}$ with a similar switch error rate of $0.3 \%$. The Canu assembly had more frequent long-range switches among haplotypes, resulting in NG50 phase blocks of $100 \mathrm{kbp}$.

Visualizing hap-mer presence in each haplotype assembly is also useful to detect overall phase consistency. When counting Col- and Cvi-specific k-mers in contigs of the TrioCanu assembly (Fig. 4c), each contig was successfully separated by haplotype as expected. That is, the Col markers were observed in the Col haplotype assembly, with almost no contaminating Cvi markers, and vice versa. The count of the found k-mers can be used as the $x$ - and $y$-axis when plotting each contig as a blob. The blob is colored by the origin of the assembly, with the size being relative to the contig size. A haplotype resolved assembly with almost no haplotype switches is expected to have all blobs close to either one of the axes, with the colors matching the corresponding haplotype of the assembly. The FALCON-Unzip alternate contigs maintain haplotype consistency (Fig. 4d), whereas the primary pseudo-haplotype contigs contain a mixture of k-mers from both haplotypes. The Canu assembly mixes haplotypes in all but the smallest contigs (Fig. 4e) and would require partitioning into primary and alternate contig sets using a post-processing tool such as purge_dups [35]. 


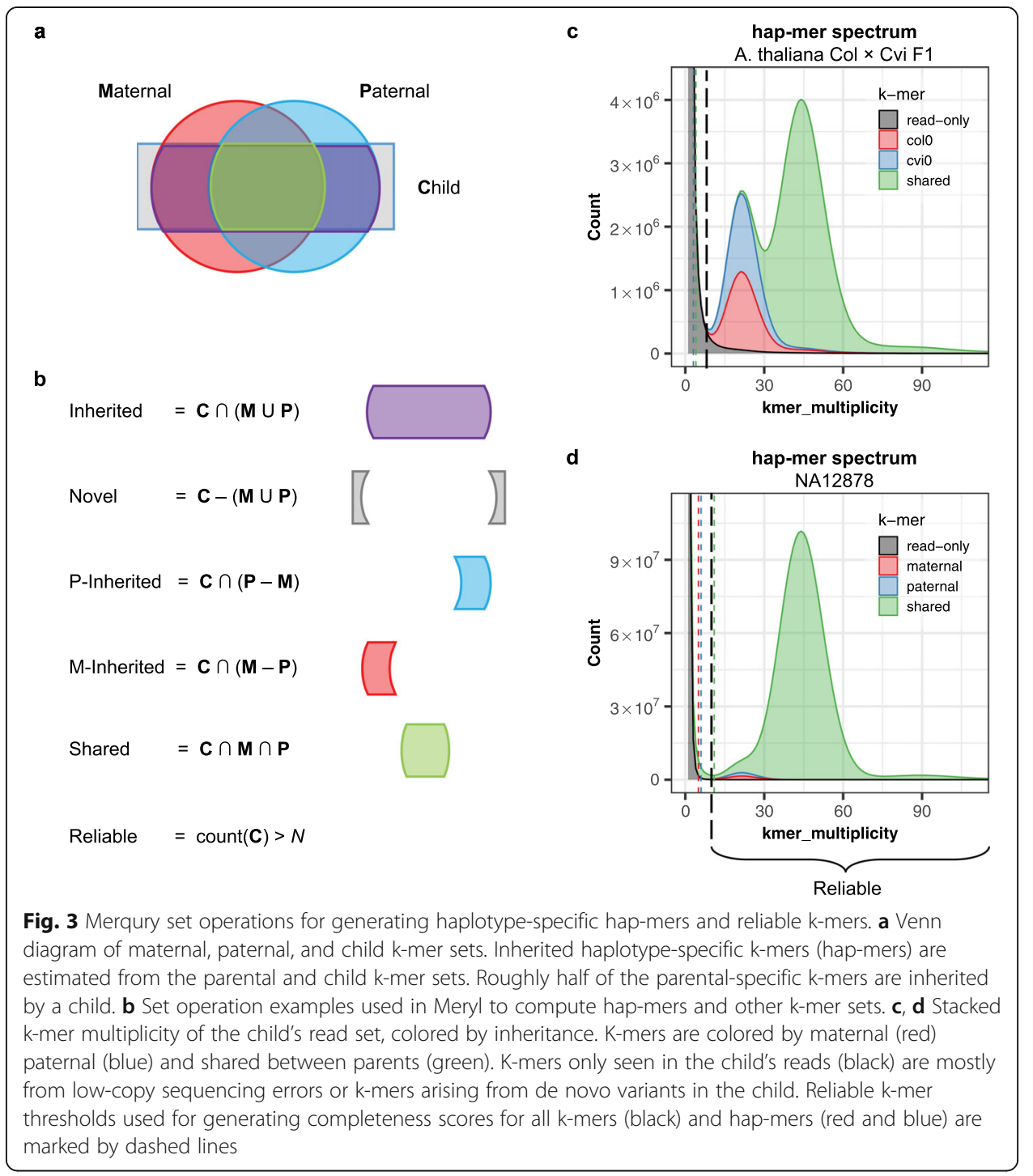

When plotting phase blocks sorted by size, the blocks originating from the wrong haplotype were very small and almost negligible in the TrioCanu assembly (Fig. 4f). In contrast, the phase blocks were highly mixed in the pseudo-haplotype assemblies, with the larger contigs being more likely to contain markers from both haplotypes (Fig. 4g, h). Plotting the contig and block sizes together shows that the trio-binned phase blocks are very similar in size to the trio-binned contigs (Fig. 4i). In comparison, the phase blocks were shorter than the contigs in the FALCON-Unzip assemblies (Fig. 4j), showing relatively good phasing performance. The phase blocks were much shorter in the Canu contigs, indicating frequent block switches between haplotypes (Fig. 4k) since Canu does not attempt to preserve long-range phasing.

Another useful feature in Merqury is that all hap-mers, erroneous k-mers, and phase blocks can be visualized as genome tracks along the assembly. Figure 5 shows an example of a 60-kbp region in the mixed-haplotype Canu assembly, where haplotypes are observed switching from Cvi (blue) to Col (red), resulting in numerous base errors (gray). This illustrates how failure to separate haplotypes can result in an increased base 


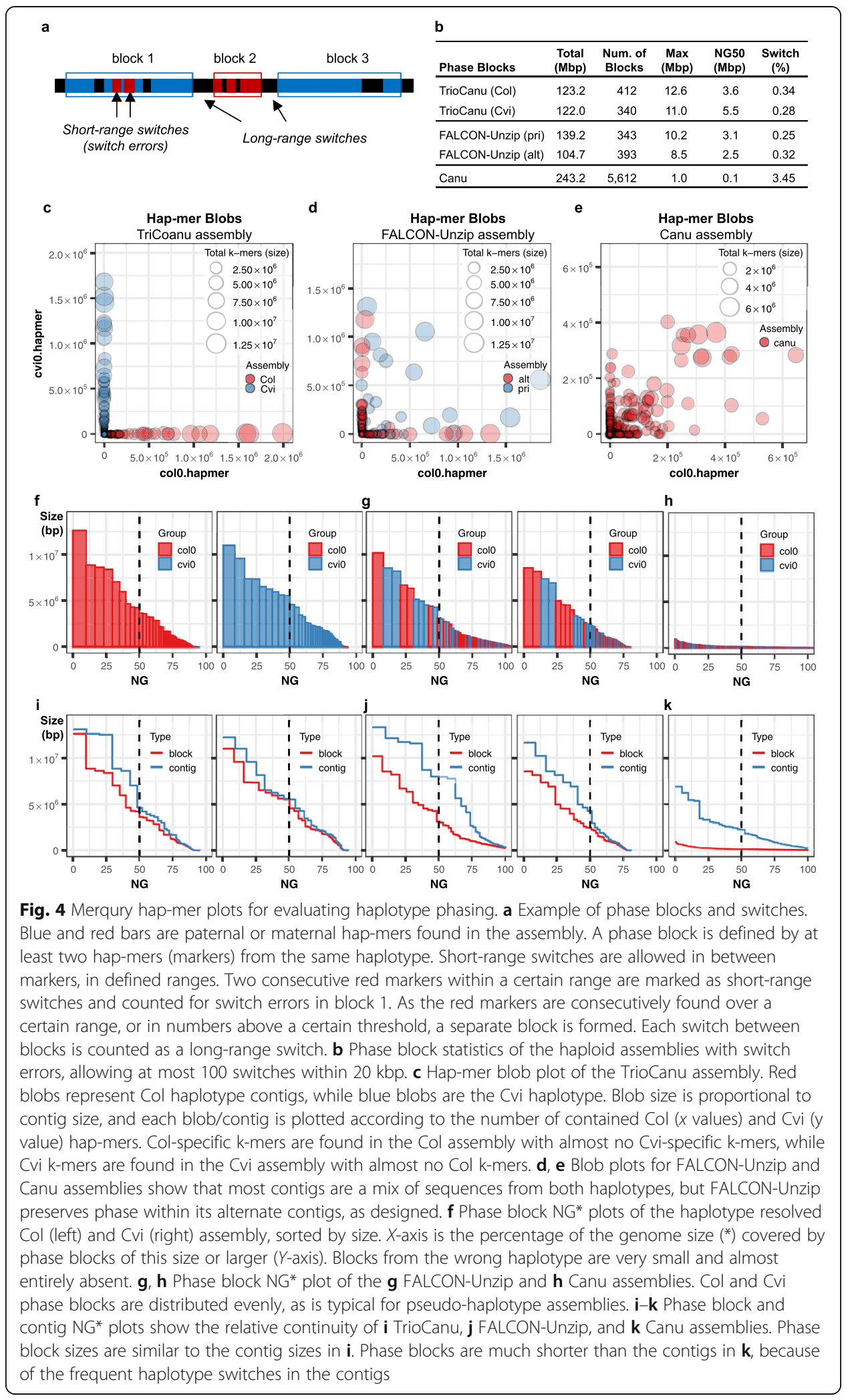




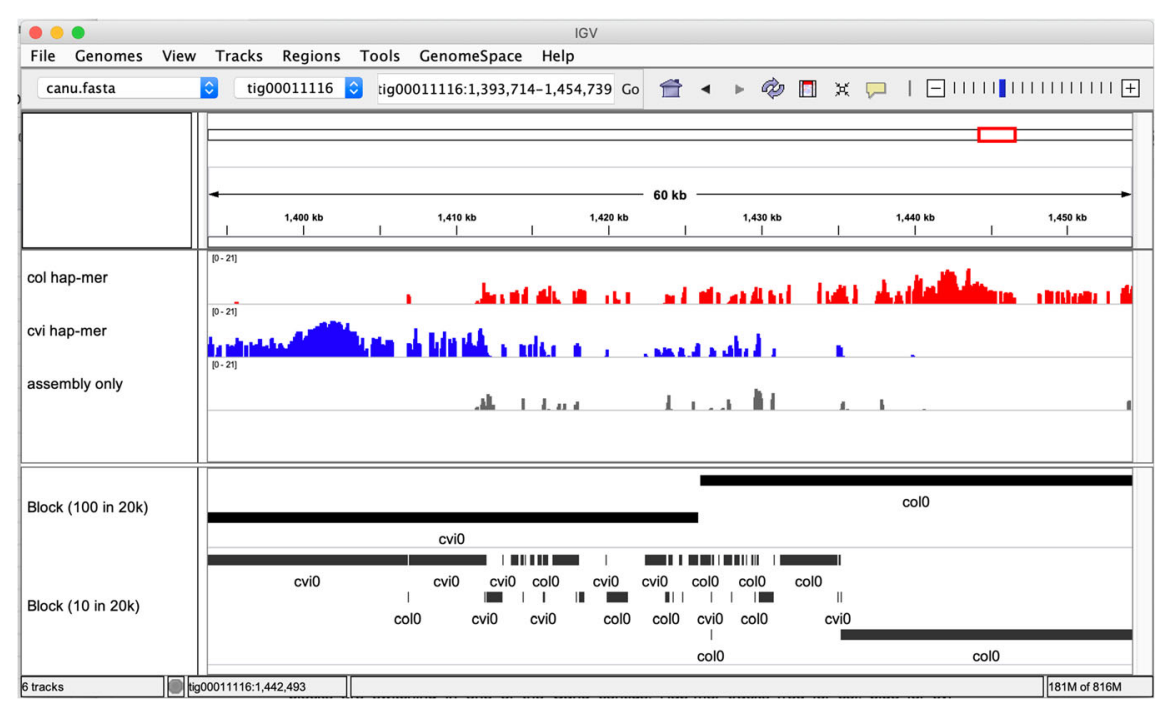

Fig. 5 Example k-mer and phasing tracks provided by Merqury. Hap-mer density is provided in tiled data format (.tdf) browsable with the Integrated Genomics Viewer (IGV) [37, 38]. This figure shows a region where haplotype blocks are switching within one of the Canu contigs. Hap-mer tracks show haplotype switches from Cvi (blue) to Col (red), along with k-mers found only in the assembly (gray), which are likely caused by erroneous consensus bases. Phase blocks (black) are shown for both relaxed (100 consecutive switches allowed per $20 \mathrm{kbp}$ ) and strict (10 per $20 \mathrm{kbp}$ ) switching thresholds

error, as the two haplotypes are improperly combined into a single consensus sequence resulting in artifactual variants. Two phase blocks (Cvi and Col) were found in this region using the default threshold of 100 consecutive k-mer switches allowed per every $20 \mathrm{kbp}$. However, phase blocks can be more stringently measured by defining the shortrange switch allowance (e.g., 10 per $20 \mathrm{kbp}$, Fig. 5, bottom track), resulting in lower NG50 phase block size (100 kbp decreases to $33 \mathrm{kbp}$ ). In contrast, the per-block switch error rate decreased from $3.4 \%$ to $0.47 \%$, making each block a more reliable haplotype. Note the per-block switch error rate is defined as the fraction of k-mer markers within a block that are assigned to the wrong haplotype, thus accounting for all short-range marker switches in a block.

\section{Benchmarking on a human genome (NA12878)}

To benchmark Merqury on a large genome, we applied it to the TrioCanu human (NA12878) assembly from Koren et al. [10] and estimated the consensus quality as Q31 for each haplotype (Table 2). The alternative variant calling approach reported 2.1 million bases of errors, resulting in a QV estimate of 34.1. However, the mappingbased approach excluded 212.2 Mbp of assembled sequence because of too few $(<3 \times)$ or too many $(>600 x)$ aligned reads. We argue that Merqury's k-mer-based approach is both more efficient and more accurate for evaluating consensus accuracy.

Merqury required only $14.9 \mathrm{CPU} \mathrm{h}(9.1 \mathrm{~h}$ for k-mer counting, $4.7 \mathrm{~h}$ for merging, $1.1 \mathrm{~h}$ for statistics) to evaluate QVs for both haplotypes. In contrast, the mapping-based QV estimate took $338.3 \mathrm{CPU} \mathrm{h}$ ( $2.2 \mathrm{~h}$ for indexing, $308 \mathrm{~h}$ for mapping, $12.6 \mathrm{~h}$ for merging, $12.8 \mathrm{~h}$ for variant calling, and $2.5 \mathrm{~h}$ for coverage calculation and QV estimates). By excluding low- and high-coverage regions of the assembly, the mapping-based approach 
ignores regions of the assembly likely to be enriched for error. For example, low coverage regions can be caused by regions of high error rates that make it difficult to map short reads. High coverage regions are typically caused by repeats that can be collapsed, and therefore incorrect, in the assembly. Thus, a substantial number of errors may be excluded from the accuracy statistics if one considers only the mappable portion of the assembly. This matches with our observation that the mapping-based estimates always overestimate QV compared to the k-mer-based approach (Table 2).

Exact k-mer counting is currently the most resource-intensive step of Merqury, requiring a maximum $21 \mathrm{~GB}$ of memory using $25.5 \mathrm{~GB}$ of disk space on NA12878 (Table 3). While this step can be parallelized across multiple nodes and cores, QV statistics can be also estimated from subsampled k-mers with lower memory and disk requirements using Mash Screen [39]. Because Merqury's QV estimation is based on Mash's k-mer containment score (Methods), the Meryl and Mash counting methods are interchangeable. In comparison to Meryl, Mash streams sequencing reads from disk and compares them against only a small subset of k-mers in the assembly. This avoids the need for a large table of k-mers but at the same time ignores copy number information. As a result, we observed that Mash QV estimates were slightly lower than exact counting (Meryl) for each haplotype, and even lower when both haplotypes were combined (Table 2). This is because the shared k-mers between the two haplotype assemblies are considered only once by Mash, resulting in an underestimate of the QV score (e.g., if a 2-copy k-mer appears in just one haplotype, it is considered "correct" by Mash). The Mash approach also cannot investigate positional base errors (Fig. 5) and many of the other analyses presented here but is provided as an alternative to Meryl for QV estimation in cases where disk and memory resources are limited.

We also compared CPU time and maximum memory used by Merqury and KAT comp to generate the same spectra-cn plots for the TrioCanu assembly of NA12878. Here, we compared Merqury and KAT comp for a single haploid assembly (maternal) and for the diploid assembly, which includes the maternal, paternal, and combined haplotype assemblies (Table 4). When run on the haploid assembly, Merqury was able to generate a spectra-cn plot and all other evaluation metrics using half the memory (21.2 GB vs $44.1 \mathrm{~GB}$ ) and comparable runtime to KAT, which generates only a spectra-

Table 3 Merqury runtime, memory, and disk requirements for QV estimation in a human genome

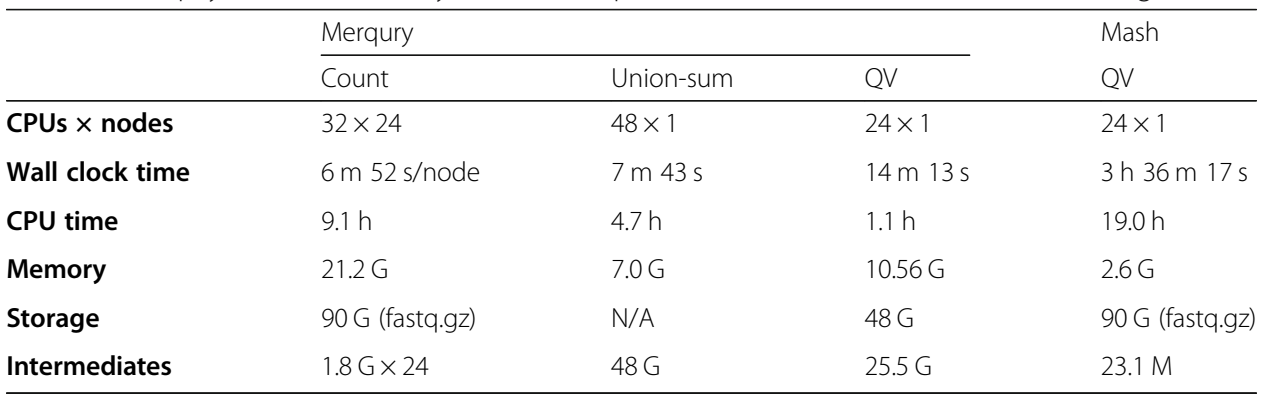

All statistics are for the diploid (maternal, paternal, and combined) assembly of the human genome NA12878. Merqury QV estimates are generated from the full k-mer databases and use exact k-mer counting, whereas Mash QV estimates are generated by streaming all reads against a MinHash sketch of the assembly using Mash Screen. Merqury's Count and Union-sum steps count all k-mers in the reads, while the QV estimation counts k-mers in the assembly and compares these to the read counts. Mash's QV estimation creates a k-mer sketch for the assembly and streams all reads against the sketch. Results are totaled over three QV operations (maternal, paternal, and combined). Runtimes were measured on Intel(R) Xeon(R) Gold 6140 CPU, with 2.30GHz. Storage requirements represent gzipped FASTQ files for counting and QV (Mash), and a binary database for QV (Meryl)

$h$ hours, $m$ minutes, $s$ seconds, $G$ gigabytes 
Table 4 Runtime and memory comparison between Merqury and KAT comp

\begin{tabular}{|c|c|c|c|c|c|c|c|c|}
\hline \multirow{3}{*}{$\begin{array}{l}\text { Assembly } \\
\text { Compute } \\
\text { Function }\end{array}$} & \multicolumn{4}{|c|}{ Haploid (maternal) } & \multicolumn{4}{|c|}{ Diploid (maternal, paternal, and combined) } \\
\hline & \multicolumn{2}{|l|}{ CPU (h) } & \multicolumn{2}{|c|}{ Max mem (GB) } & \multicolumn{2}{|l|}{ CPU (h) } & \multicolumn{2}{|c|}{ Max mem (GB) } \\
\hline & Merqury & KAT & Merqury & KAT & Merqury & KAT & Merqury & KAT \\
\hline Count $k$-mers in reads & 13.8 & 22.4 & 21.2 & 44.1 & 13.8 & 63.0 & 21.2 & 44.1 \\
\hline Spectra-cn & 3.9 & & 10.1 & & 9.7 & & 10.6 & \\
\hline Spectra-asm, QV, Completeness & & - & & - & & - & & - \\
\hline Spectra-hap & 2.0 & - & 1.5 & - & 3.4 & - & 3.2 & - \\
\hline Blobs, phase block, switch error & 2.8 & - & 10.3 & - & 5.6 & - & 8.1 & - \\
\hline Total & 22.5 & 22.4 & 21.2 & 44.1 & 32.5 & 63.0 & 21.2 & 44.1 \\
\hline
\end{tabular}

All statistics are from the haploid and diploid human genome NA12878 assembly. KAT comp was run once and three times to generate the equivalent spectra-cn plots generated with Merqury. In addition to the spectra-cn plots, Merqury simultaneously generates spectra-asm, QV and k-mer completeness statistics, which are not available in KAT. Runtimes were measured on Intel(R) Xeon(R) Gold $6140 \mathrm{CPU}$, with $2.30 \mathrm{GHz}$, using a maximum of 24 cores. CPU time is measuring runtime assuming a single core for comparison. Maximum memory footprint is reported in Max mem

cn plot (22.5 CPU h vs 22.4 CPU h). Merqury runtime includes the construction of a k-mer count database of the reads, which must only be generated once. Thus, when using a pre-built database, the run time requirement drops by more than half (8.7 CPU $\mathrm{h}$ per assembly). In comparison, KAT must recount k-mers in the reads for each spectracn plot, and as a result, the runtime of KAT triples to $63.0 \mathrm{CPU} h$ when evaluating the diploid assembly. Merqury utilizes efficient set operations on k-mer databases, allowing it to count k-mers in each haploid assembly only once and merges them internally to produce the k-mers of the combined assembly. The diploid assembly can be analyzed in 32.5 CPU h, or 19.7 CPU h with a pre-built database, including the generation of Merqury's additional quality and phasing metrics.

Next, we evaluated k-mers for NA12878. The hap-mer spectrum of NA12878 revealed a higher fraction of shared k-mers in the 1-copy peak (Fig. 3d), indicating that some heterozygous variants in the child are shared by both parents. We do not see such a strong effect in A. thaliana, because the parents were heavily inbred and contained few heterozygous variants of their own. In contrast, the A. thaliana F1 hybrid was deliberately outbred, which is evident by the dramatically taller 1-copy peak versus NA12878 (0.99 vs. $0.12 \%$ heterozygosity).

Haplotype-specific k-mers are convenient to obtain haplotype precision (PPV) and recall (completeness) statistics based on how many of the expected parental k-mers are observed in the child's haplotype-resolved diploid assembly (Fig. 6). To demonstrate, we built genomic k-mer databases for NA12878 and her parents, totaling 18.4 and 19.9 million inherited hap-mers for the paternal and maternal haplotypes, respectively. When comparing to the haplotype-resolved assemblies, the maternal haplotype assembly recovered $90.7 \%$ of the maternal hap-mers (Table 2 and Fig. 6a), and the paternal assembly recovered $91.0 \%$ of the paternal hap-mers (Table 2 and Fig. 6b). Likewise, by considering the other haplotype's markers as false positives (i.e., paternal hap-mers found in the maternal assembly), the precision of the maternal and paternal assemblies was 99.1 and 98.8\%, respectively, with only 160 200 markers appearing in the incorrect haplotype (Table 2, PPV values). This evaluation excludes all k-mers found only in the assembly (errors), which if considered false positives, would further lower the precision.

To compare Merqury's results with an alternative approach, we considered an Illumina-based platinum callset for NA12878 [27] that includes 3.4 million 


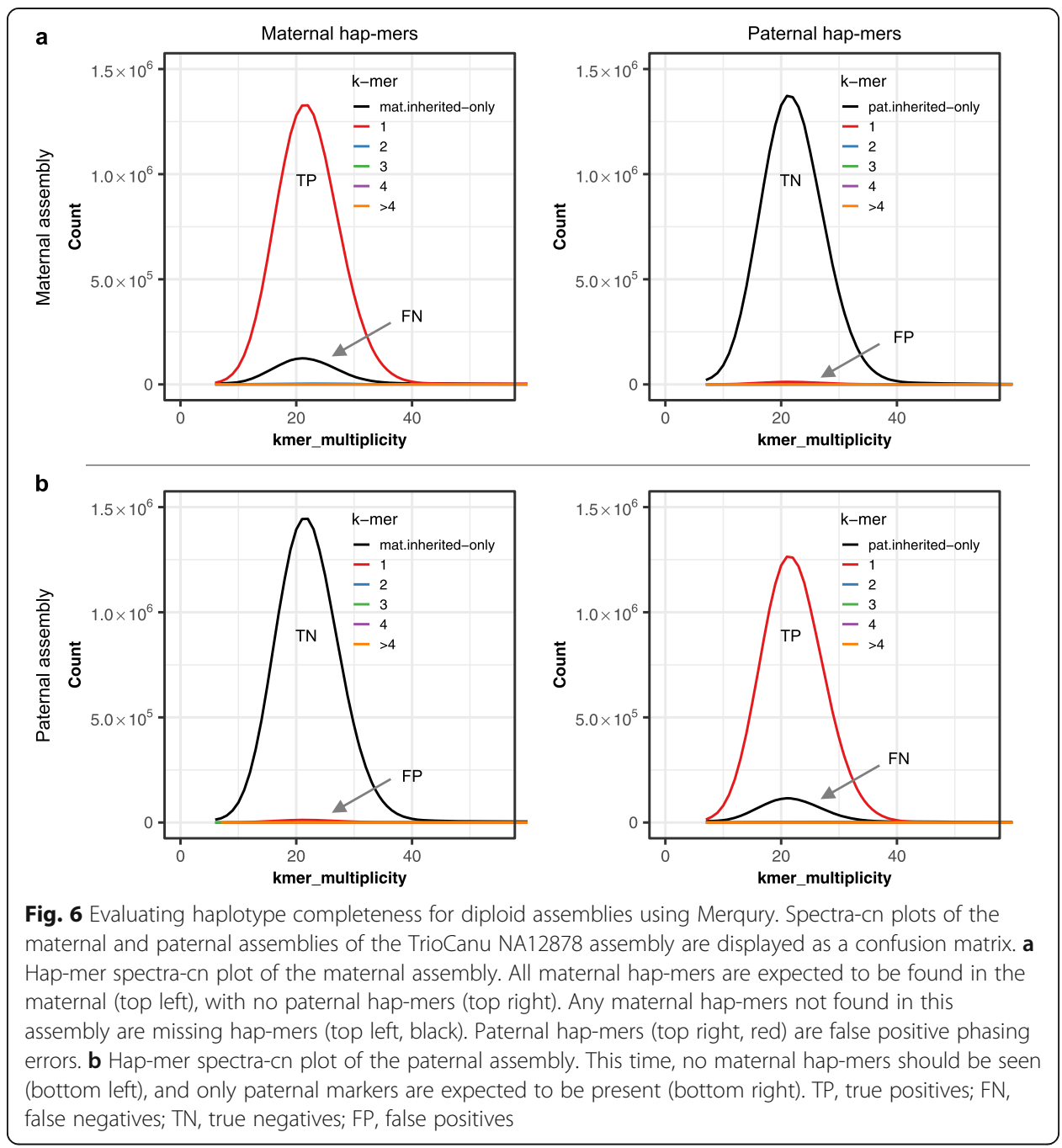

heterozygous SNPs in regions where both haplotype assemblies align to the reference (hg38). Calling SNPs directly from the haplotype assemblies against hg38 recovered 3.2 million variants, or $93.5 \%$ sensitivity, which is slightly higher than the Merqury based estimate of $\sim 91 \%$. This discrepancy is likely due to Merqury's ability to measure complex regions of the assembly not easily measurable by a mapping-based analysis. In contrast, the SNP-based measurement of precision was only $86.1 \%$, likely due to the low base accuracy $(\mathrm{Q} 31)$ of the assemblies, where consensus errors are called as falsepositive heterozygous SNPs. Thus, it is important to consider both the k-mer QV and precision estimates when evaluating the accuracy of a diploid assembly.

\section{Discussion}

We have developed Merqury, a reference-free assembly evaluation toolkit based on efficient k-mer-based methods. Merqury builds upon the k-mer spectra ideas of Mapleson et al. [17] and introduces novel methods and plots for measuring assembly quality (QV), completeness, and phasing. Using k-mer count spectra, Merqury can reveal copy-number errors in an assembly and accurately measure both assembly completeness and consensus quality. When parental k-mers are available, Merqury can also 
measure phasing accuracy and haplotype completeness. In addition to validation statistics, Merqury provides a number of graphs for interpreting assembly quality. In the process of developing Merqury, we have also extended Meryl with generalized k-mer counting, querying, and set operations that will be useful for other k-mer-based analyses (Fig. 3b).

Merqury is able to evaluate assemblies from any sequencing technology and works best when high-accuracy sequencing reads are available from the assembled individual and its parents. This read set serves as an independent validation of the assembly, which is typically based on less-accurate, long-read sequencing. If high-accuracy reads are not available from the assembled individual, read sets from the parents can be used as a replacement for measuring quality values. This assumes all k-mers in the child are found in the parental genomes, ignoring the small fraction of k-mers from de novo mutations in the child. Although we currently recommend using Illumina data for the k-mer-based validation, it may require special library preparation to minimize sequencing biases [40-42]. We note that Merqury's methods are general and would be compatible with any reasonable high-accuracy, high-throughput sequencing technology.

Currently, Merqury does not explicitly validate the structural accuracy of an assembly, which is another important factor of assembly quality [32]. Although certain misassemblies, such as the systematic collapse of repeats, would be visible from the spectra-cn plots, other types of structural mis-assemblies, such as inversions, could go unnoticed. This is one drawback of Merqury's k-mer-centric design. However, we have observed that regions of mis-assembly often correlate with a decrease in consensus quality, similar to the haplotype switch example shown in Fig. 5. This information can be used to draw attention to unreliable regions of the assembly that can be later validated using orthogonal approaches.

Hap-mers are currently computed by a simple set operation, similar to trio-binning [10]. A higher portion of hap-mers are identifiable when the parents are divergent, with minimal shared ancestry. Our hap-mer spectrum plots (Fig. 3c, d) show the 1-copy haplotype markers that are specific to each parent, which may not be as prevalent for genomes of low heterozygosity, such as humans. Merqury is also extensible to polyploid genomes for most of the evaluations. However, for the phasing measurements, Merqury currently reports at most two parental (or ancestral) haplotypes. In the case of a polyploid genome, Merqury's reported phase blocks would reflect inheritance from a single parent, but not necessarily the correct phasing of homologous chromosomes (e.g., two homologous chromosomes inherited from the same parent would be indistinguishable). To accurately measure the phasing of homologous chromosomes in a polyploid genome would require a different method for identifying hap-mers that does not rely solely on parental inheritance. To support alternative k-mer classification methods (e.g., Hi-C, Strand-seq, linked-reads), Merqury is designed to receive any pre-computed hap-mer set as input.

We argue that Merqury's k-mer-based method provides better haplotype completeness estimates, because it does not rely on a reference genome. Mapping to a reference can be biased by mis-mapping to repetitive or low-quality regions of the assembly. Moreover, k-mers naturally capture heterozygous insertion and deletion variants and are thus immune to the problems of calling these types of variants with a reference mapping approach. For example, consortiums such as the GA4GH exclude all variant 
calls within complex, repetitive regions of the genome [26]. In contrast, k-mers inherently capture genetic context, regardless of the structural complexity surrounding them in the genome. Moreover, k-mers are especially robust for evaluating sequences in highly diverged sequences, where mapping-based approaches cannot map reads to call variants.

Lastly, Merqury provides an efficient way of determining phase blocks in diploid assemblies. In the past, phase blocks were defined based on heterozygous SNPs, measured by aligning each haplotype to one another [9] or by mapping to a reference genome [8]. These alignment-based approaches may not consider the full genome when the identity between the two haplotypes is lower than the alignment threshold, or the alignment is confused by genomic repeats. Moreover, a reference genome may not represent the entire haplotype of an assembled individual, thereby omitting haplotypespecific sequences from the analysis. The phase blocks measured by Merqury are generated regardless of the haplotype being assembled and provide more reliable phasing information for allele-specific studies.

\section{Conclusions}

Merqury provides a suite of efficient methods for assessing the quality, completeness, and phasing of genome assemblies using a reference-free, k-mer-based approach. Merqury extends the spectra-cn functionality of KAT and introduces novel features such as spectra-asm, spectra-hap, and blob plots; assembly QV and completeness measurements, and the first reference-free approach for measuring assembly phase blocks using parental k-mers. The included Meryl k-mer counter also provides fast and flexible methods for k-mer set manipulation. Compared to traditional assembly metrics, such as N50 contig size, Merqury provides a much broader evaluation of assembly quality and we recommend reporting these metrics along with any new genome assembly.

\section{Methods}

\section{Counting k-mers with Meryl}

Meryl is a tool for counting and working with sets of k-mers that was originally developed for use in the Celera Assembler [43] and has since been migrated and maintained as part of Canu [33]. Here we have improved Meryl's efficiency and extended it to support a variety of functions useful for k-mer-based assembly validation. A set of k-mers and their associated counts is termed a k-mer database. The count is the number of times a k-mer occurs in some collection of sequences. The k-mer database is stored in sorted order, similar to words in a dictionary. Meryl comprises three modules: one for generating kmer databases, one for filtering and combining databases, and one for searching databases. All Meryl operations are exact (i.e., do not result in false positives or negatives).

The counting module uses two different algorithms: one for k-mers up to size 16 and the other for k-mers up to size 64. For small k-mers, Meryl directly counts the number of times each k-mer occurs in the input sequences. An array of $4^{k} 16$-bit integers is allocated. Each k-mer is converted to an integer index into the array, and the cell for that $\mathrm{k}$-mer is incremented. When any cell exceeds the maximum possible value that can be represented, the width of the array is extended by allocating a supplementary array of $4^{\mathrm{k}}$ bits. For large k-mers, Meryl generates lists of all the k-mers present in the input 
sequences, sorts each list, then scans each to determine how many times a specific kmer occurs. Each k-mer is split into a prefix and a suffix. The prefix is used to select a list, and only the suffix is added. A trade-off is made between a small prefix (which would result in only a few lists, each storing many suffixes) and a large prefix (which would result in many lists). As we do not know how big each list will be, the lists must be able to grow as needed. Each list is therefore an array of memory blocks where each block can store a few thousand k-mers. While counting, the memory usage of the lists is tracked, and if a user-supplied memory limit is reached, the lists are sorted, k-mers are counted, and output written to an intermediate database. After all k-mers are processed, the intermediate, sorted databases are efficiently merged into one.

With one or more databases on disk, Meryl can filter or combine k-mers to create new databases. Each database is stored in 64 independent pieces, and each piece can be processed in parallel. Within each piece, k-mers are in the same order; thus, all set operations require linear time and constant space. Meryl can filter a database by count (e.g., less-than, greater-than or equal-to some user-supplied constant), or by a fraction of distinct k-mers in a database (e.g., the most common $5 \%$ of the k-mers). It can modify the count of every k-mer in a database by a constant (e.g., add 1, subtract 1 , multiply by 2). Meryl can also output the union or intersection of multiple databases, setting the count of a k-mer to the minimum, maximum, sum of all copies of the k-mer, or as the count of the first database. It can output the difference of databases (e.g., the k-mer occurs only in the first database) or the symmetric difference (e.g., the k-mer occurs in exactly one database). Conveniently, any number of these operations can be combined into one command, using a reverse-polish-notation inspired format. The following example invocations are used in Merqury:

1. Write the k-mers that occur in both db1.meryl and db2.meryl to db3. meryl. Set the count of each output k-mer to the sum of the counts in the input k-mers:

meryl union-sum db1.meryl db2 . meryl output db3 . meryl

2. Output k-mers that occur in both db4 . meryl and db5.meryl, additionally requiring the k-mer in db5.meryl to be unique. The count of the output k-kmer is set to the count of the k-mer in the first input to the intersect operation,

namely db4 .meryl:

meryl output db6.meryl $\backslash$

intersect $\backslash$

$\mathrm{db} 4$.meryl $\backslash$

[ equal-to 1 db5.meryl ]

3. For each k-mer in asm. fasta, output the (0-based) coordinate of the kmer in the sequence, the forward and reverse k-mer sequences, and the count of the kmer in db7.meryl:

meryl-lookup -dump-sequence asm.fasta -mers db7.meryl

Meryl includes a C++ API to extend its functionality. For example, random lookups can be added using either the simple existence of a k-mer in a database or the count associated with a k-mer. On the command line, lookups can return the number of kmers a sequence shares with a database, a list of each k-mer in a sequence annotated 
with the count the k-mer has in a database, or a filtered list of input sequences based on the presence or absence of $k$-mers in the database.

\section{Evaluating assemblies with Merqury Copy number spectrum (spectra-cn plot)}

Given a genome size $G$ and tolerable collision rate $p$, an appropriate $k$ can be computed as $k=\log _{4}(G(1-p) / p)$ [44]. However, alternative values of $k$ may be desirable for different scenarios, e.g., increasing $k$ has the effect of increasing the relative fraction of 1copy k-mers, which may be useful for genomes with low heterozygosity, but this also increases the fraction of erroneous k-mers. Decreasing $k$ reduces the fraction of erroneous k-mers but increases the fraction of repetitive k-mers. Once an appropriate size of $k$ is determined (typically 18 21), we count the canonical k-mers observed in the assembly and in the accurate, whole-genome read set. A typical k-mer spectrum for a heterozygous diploid genome consists of two primary peaks, one representing k-mers that are 1-copy in the diploid genome (heterozygous, on a single haplotype) and one representing those that are 2-copy in the diploid genome (homozygous, on both haplotypes or two copies on one haplotype) (Fig. 1a). The 2-copy k-mers appear with a frequency approximately equal to the average depth of sequencing coverage, where the 1-copy k-mers appear with frequency approximately equal to half the sequencing coverage. If a genome is entirely homozygous, only the 2-copy peak may appear, and if the genome is extremely heterozygous, only the 1-copy peak may appear. With sufficient sequencing coverage (to separate the peaks along the axis), and a proper choice of $k$, both peaks are visible for most genomes. Using the multiplicity of the k-mer counts, and modeling the k-mer survival rate (i.e., how many k-mers are unaffected by sequencing error), it is possible to predict the size and repeat content of a genome from the kmer spectrum alone [36].

The spectra-cn plot was introduced by Mapleson et al. [17], which colors k-mers of the read set by their copy numbers in the assembly. In addition to the original stacked version of the spectra-cn plot (Fig. 1b), we provide additional options to plot the unstacked copy number spectrum (Fig. 1c). We have found this style more useful for visually detecting abnormal k-mer copy numbers and their distribution in an assembly.

\section{Assembly spectrum (spectra-asm plot)}

Similar to the spectra-cn analysis, we can color each k-mer in the read set by the assembly in which it is found. This becomes useful when two haploid assemblies are evaluated. This way, we can detect k-mers that are present only in one assembly, k-mers shared in both assemblies, and k-mers not present in the assembly and only found in the read set (Fig. 2).

\section{K-mer completeness}

We define a "reliable k-mer" as a k-mer that is truly in the genome and unlikely to be caused by sequencing error. With exact k-mer counts, it is easy to filter out low-copy $\mathrm{k}$-mers that are likely to represent sequencing errors. We use the same strategy as Koren et al. [10] to find the cutoff. In brief, we take the histogram of the k-mer counts and set the multiplicity (number of times we see the k-mer in the read set) as $x$ and 
counts (number of k-mers with $x$ multiplicity) as $y$. When differentiating the histogram, we compute the slopes and the first k-mer multiplicity with a positive slope defines the reliable k-mer threshold. Examples of these cutoffs are shown as dashed lines in Fig. 3c, $\mathrm{d}$. The k-mer completeness is calculated as the fraction of reliable k-mers in the read set that are also found in the assembly. For repetitive genomes, erroneous read set kmers can sometimes appear above this threshold due to recurring errors in high-copy repeat families, but this is rare.

\section{Consensus quality (QV) estimation}

We can also use k-mers to estimate the frequency of consensus errors in the assembly. We use a binomial model of k-mer survival and assume all k-mers in the assembly should be found at least once in the read set. Here, we use the containment score from Mash Screen [39] to estimate consensus accuracy. In brief, we estimate the probability $P$ that a base in the assembly is correct as:

$$
P=\left(\frac{K_{\text {shared }}}{K_{\text {total }}}\right)^{\frac{1}{k}}
$$

where the $K_{\text {total }}$ is the total number of k-mers found in an assembly and $K_{\text {shared }}$ are the number of shared k-mers between the assembly and the read set. If the read set is assumed to completely cover the genome, any k-mer found only in the assembly $\left(K_{\text {asm }}=\right.$ $\left.K_{\text {total }}-K_{\text {shared }}\right)$ likely reflects a base error in the assembly consensus. Hence, the error rate $E$ can be defined as:

$$
E=1-P=1-\left(1-\frac{K_{\text {asm }}}{K_{\text {total }}}\right)^{\frac{1}{\mathrm{k}}}
$$

Using this formula, the widely used Phred [45] quality score (often denoted as QV) can be computed by treating the $E$ as base error probability:

$$
\mathrm{QV}=-10 \log _{10} E
$$

Note that this QV estimate depends on the coverage and quality of the read set. The assembly QV will be underestimated if the read set does not contain all true k-mers of the genome due to low or heavily biased sequencing coverage. Conversely, the QV may be overestimated if the read set contains a high proportion of false k-mers (e.g., due to the combination of extreme coverage and sequencing error). Lastly, the k-mer survival model assumes both k-mers and errors are independent, but k-mers overlap one another and errors tend to cluster together. As a result, the choice of $k$ also affects the QV estimates, with $k \approx 21$ recommended by Mash based on empirical testing.

\section{Positions of k-mers for mis-assembly detection}

Merqury reports the positions of the k-mers found only in an assembly for further investigation in .bed and .tdf formats that can be loaded into most genome browsers. In addition, the k-mers found in unexpected copy numbers (i.e., false duplications) are also provided as .bed and .tdf files. The format details are described at:

https://genome.ucsc.edu/FAQ/FAQformat.html\#format1

https://github.com/igvteam/igv/wiki/TDF-Format 


\section{Evaluating phasing completeness with parental genomes Haplotype-specific $k$-mers (hap-mers)}

Parental haplotype markers can be obtained directly from the parental or ancestral genomes [10]. In brief, distinct k-mers found in only one parent are collected, and the erroneous low-frequency k-mers are filtered out. This filtering strategy relies on the kmer count histogram, where the cutoff for identifying reliable k-mers is computed as described above. When the child's short-read data is also available, the inherited haplotype-specific markers can be obtained by intersecting the child's k-mers with the parental marker sets. This time, we keep the k-mer counts from the child's reads for further ploidy estimation (Fig. 3).

\section{Hap-mer blob plot}

To get a global visual representation of the phasing completeness on assembled sequences, we can count the total number of hap-mers found on each contig or scaffold (Fig. 4c, d). Here, each axis becomes the number of hap-mers found in a sequence entry (contig or scaffold). Each circle (blob) represents a sequence, the size being relative to the length of the sequence. Sequence bubbles near the diagonal represent mixtures of markers from both haplotypes, while bubbles closer to a haplotype axis are predominately comprised of markers from a single haplotype.

\section{Phase block statistics and switch error}

Whenever a hap-mer switch occurs, Merqury can flag that position in the assembly and output a haplotype block report. This feature is useful for identifying phase blocks from a partially or completely phased assembly such as FALCON-Unzip [9], FALCONPhase [12], or Supernova2 [4]. Merqury defines a phase block as a continuous sequence with at least two hap-mers originating from the same haplotype (Fig. 4a). Usually, short-range switches are caused by consensus, rather than phasing, errors. By default, Merqury allows at most 100 hap-mer switches per $20 \mathrm{kbp}$ as short range switches within a phase block. Each unexpected hap-mer found will be counted as a switch error but will not necessarily terminate the phase block. Ideally, when no switches are found, the phase block N50 will be the same as the scaffold N50 and the sum of the phase blocks will be identical to the assembled sequences. In reality, a scaffold often does not end with a haplotype-specific sequence, so the total phase block length is shorter. Triobinned or haplotype-resolved assemblies are a special case, where the entire haplotype assembly is essentially a single block. Still, in this case, hap-mers from the other haplotype can be counted as switch errors. Merqury also provides an option to restrict phase blocks to contigs and break the blocks at any gap. At the end, Merqury reports the number of switches and total hap-mers on each block along with the switch error rate in order to identify blocks with more frequent switch errors.

\section{Hap-mer copy number spectrum}

The total k-mer spectrum of the assembled individual is also useful for tracking the fates of haplotype-specific k-mers in a diploid assembly. Similar to the overall copy number analysis performed with spectra-cn plots, we can count k-mers in each haplotype assembly and estimate the completeness of haplotype-specific assembled bases 
compared to the hap-mer sets. For example, by plotting separate histograms for hapmers of different copy numbers in the assembly (Fig. 6), we can identify whether the assembly is artificially collapsing or duplicating sequence in each haplotype. If hap-mers appear over (or under) represented by the assembly relative to the read set, it is an indicator of artifactual duplication (or absence) of haplotype-specific sequence. When evaluating a pseudo-haplotype assembly, which is designed to collapse or pick one haplotype as much as possible, we can count the number of hap-mers present in the child's read set but not present in the pseudo-haplotype assembly and use this to quantify the amount of under-represented haplotype sequence. These missing hap-mers could then be used to identify a set of alternative haplotype reads that were incorrectly excluded from the assembly.

\section{Assemblies}

All TrioCanu assemblies were downloaded from Koren et al. [10], available at https:// obj.umiacs.umd.edu/marbl_publications/triobinning/index.html. The A. thaliana F1 FALCON-Unzip assembly was obtained from Chin et al. [9]. We generated a Canu assembly to show a typical example of an interim mixed-haplotype assembly that has not been polished or purged of haplotypic duplications. The same Pacific Bioscience reads were used for all three assemblies, obtained from Chin et al. [9].

The Canu assembly was generated with Canu 1.9 release version using the following command:

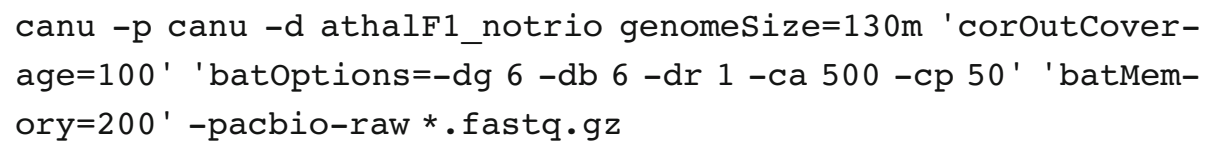

\section{Haplotype specific k-mers (hap-mers) for A. thaliana F1 and NA12878}

Appropriate size of $\mathrm{k}$ was obtained as $\mathrm{k}=18$ for the A. thaliana F1 with 130 260 Mbp genome size and $k=21$ for NA12878 with 3.2 6.4 Gbp genome size using \$MERQURY/ best_k.sh.

As parental Illumina sequencing was not available for this F1, the parental genome assemblies from Chin et al. [9] were used to obtain parental specific k-mers. Each assembly from the inbred Col-0 and Cvi-0 lines were downloaded from:

https://downloads.pacbcloud.com/public/dataset/PhasedDiploidAsmPaperData/FUN ZIP-PhasedDiploidAssemblies.tgz.

Meryl databases for the parental strains were built directly with meryl count $k=18$ output \$hap.meryl \$hap. fasta for each haplotype assembly.

The parental Illumina whole-genome sequencing sets for NA12878 were downloaded from the high coverage dataset of the 1000 Genomes Project (NA12891 and NA12892) and combined with Illumina Platinum Genomes Project data from PRJEB3381. Illumina whole-genome sequencing of NA12878 was downloaded from PRJEB3381.

All Meryl databases from sequencing read sets were built with \$MERQURY/_submit_build.sh. Once the k-mer databases were built, inherited hap-mers were obtained with \$MERQURY/trio/hapmers.sh. 


\section{Merqury on all assemblies}

Merqury was run for the A. thaliana F1 and NA12878 with the following command line for diploid assemblies, where \$hap1 and \$hap2 are maternal (mat) and paternal (pat) for the TrioCanu assemblies, and primary (pri) and alternate (alt) for FALCONUnzip assemblies.

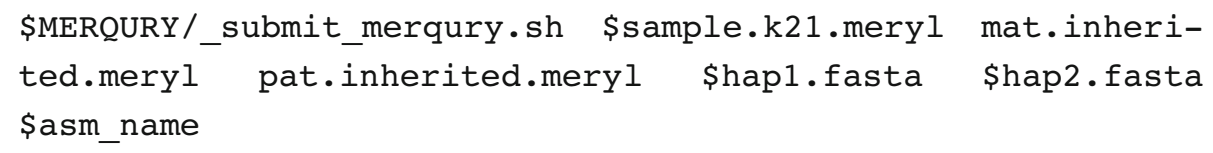

The A. thaliana F1 Canu assembly was run with:

\$MERQURY/_submit_merqury.sh \$sample.k21.meryl mat.inherited.meryl pat.inherited.meryl canu.contigs.fasta Canu

BUSCO

BUSCO v3 was run on using embryophyta_odb9 for the A. thaliana Canu assembly and the combined $\mathrm{Col}$ and Cvi TrioCanu assembly with the following commands:

python run_BUSCO.py -i asm.fasta -o SAMPLE - l embryophyta_odb9

-m genome -c 16 -sp arabidopsis

For NA12878, BUSCO was run in the same way, using mammalia_odb9 for the combined TrioCanu maternal and paternal assembly. BUSCO scores for each haplotype of $A$. thaliana F1 and NA12878 were obtained from Koren et al. [10] Supplementary Table 2.

\section{QV estimates}

CPU time, memory consumption, and disk usage were measured for generating QVs on each haploid assemblies and the combined diploid assembly. A Intel(R) Xeon(R) Gold 6140 CPU @ $2.30 \mathrm{GHz}$ node was used allowing up to 24 CPUs. Detailed node information is available at https://hpc.nih.gov.

\section{Meryl-based QV}

Meryl-based QV estimation to benchmark computing resources was evaluated for the counting (count), merging (union-sum), and QV steps with the following command:

\section{\$MERQURY/eval/qv.sh NA12878.k21.meryl mat.fasta pat.fasta} meryl_qv

This generates Meryl databases for mat.fasta and pat.fasta, does a union for the databases, and generates QV scores for all three combinations (maternal, paternal, and both).

\section{Mash-based QV}

Mash-based QV estimation was performed using sketch size of 1000000 with the same k-mer size of 21 : 
mash sketch -s $1000000-\mathrm{k}$ \$k \$asm

mash screen -p \$cpus \$asm.msh 'cat \$input_fofn $\mid$ tr ' $\backslash n$ ' ' '>> \$name.msh.idy

cat \$name.msh.idy | awk -v name=\$name ' pprint name" $\backslash t " \$ 2 " \backslash t "-$

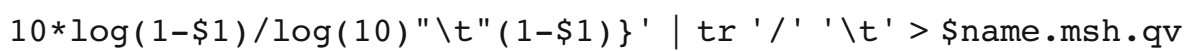

\section{Mapping-based QV}

The Illumina WGS reads used to build the Meryl database were aligned to both mat.fasta and pat.fasta using BWA [46]. Base pair errors were called using FreeBayes [47] v1.3.1 --skip-coverage 600, skipping variant calling in regions with $>600 \times$ read depth to help prevent unnecessary computing on high coverage regions that violate the diploid assumption. The exact commands used were:

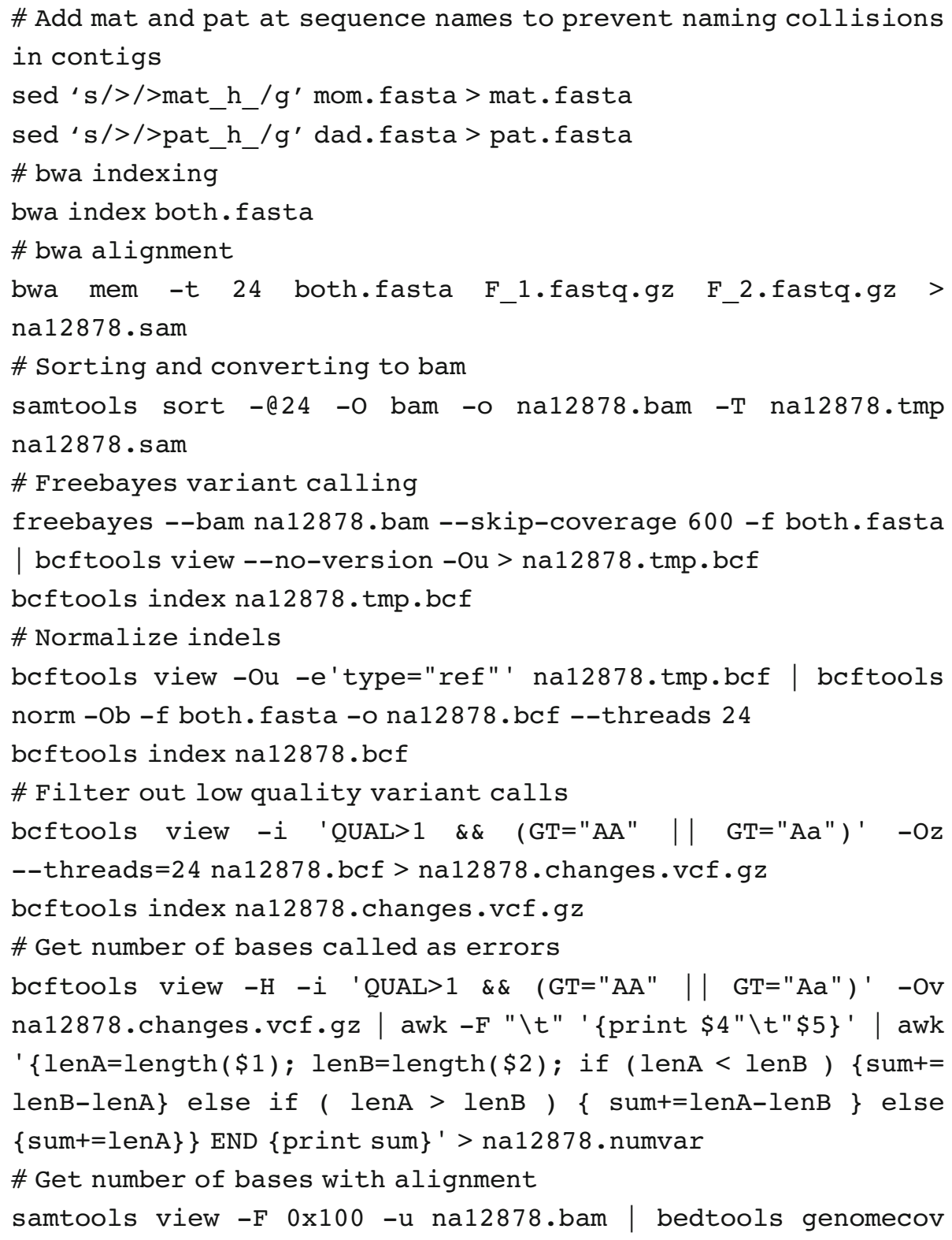




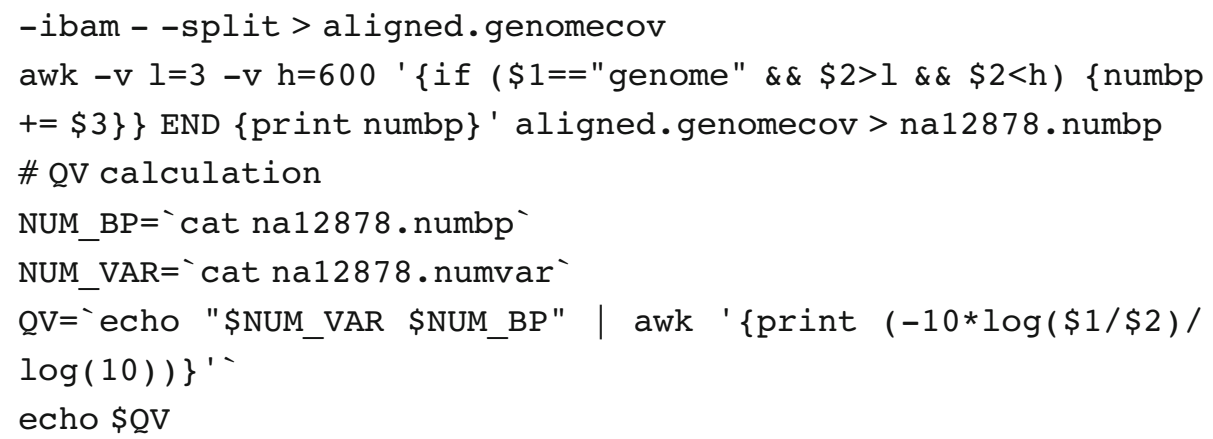

This pipeline is used in the Vertebrate Genomes Project, and the code used is available from https:/github.com/VGP/vgp-assembly/tree/master/pipeline/ under the "bwa, " "freebayes-polish," and "qv" directories.

\section{Comparison to KAT}

KAT comp (v2.4.1) was run three times using $k=21$ to generate the equivalent spectracn plots generated by Merqury. For consistency, both Merqury and KAT were run using at most 24 threads. Unlike Merqury, KAT must recount k-mers for each spectracn plot (https://kat.readthedocs.io/en/latest/faq.html\#should-i-dump-jellyfish-hashesto-disk). The following command was executed three times for the maternal, paternal, and the combined assembly:

$$
\text { kat comp -o \$out -t } 24-\mathrm{m} 21-\mathrm{h} \text { 'F_1.fastq.gz F_2.fastq.gz' \$asm }
$$

\section{Supplementary information}

Supplementary information accompanies this paper at https://doi.org/10.1186/s13059-020-02134-9.

Additional file 1. Review history.

\section{Acknowledgements}

The authors would like to thank the members of the Vertebrate Genomes Project assembly working group for helpful discussions, including Marcela Uliano da Silva and Giulio Formenti. We also would like to thank Bernardo Clavijo of the Earlham Institute for his very insightful feedback in response to a preprint of this manuscript. This research utilized the computational resources of the NIH HPC Biowulf cluster (https://hpc.nih.gov).

Review history

The review history is available as Additional file 1.

\section{Peer review information}

Barbara Cheifet was the primary editor on this article and managed its editorial process and peer review in collaboration with the rest of the editorial team.

\section{Authors' contributions}

A.R. conceived the project and implemented Merqury. B.P.W. designed and implemented Meryl. A.R., B.P.W., S.K., and A.M.P. designed the methods. A.R. and S.K. performed analyses. A.R. and A.M.P. coordinated the project. All of the authors wrote the manuscript and approved the final manuscript.

\section{Funding}

This research was supported by the Intramural Research Program of the National Human Genome Research Institute, National Institutes of Health.

\section{Availability of data and materials}

Merqury and Meryl are conceived as "United States Government Work" and are under public domain according to the terms of the United States Copyright Act. It was written as part of the authors' official duties for the US government and thus cannot be copyrighted. This software is freely available to the public for use without a copyright notice. Restrictions cannot be placed on its present or future use. Merqury is available under public domain at GitHub repository, https://github.com/marbl/merqury [48]. Meryl is also available under public domain unless otherwise stated 
as GPL-2.0 in the source code at GitHub repository, https://github.com/marbl/meryl [49]. Merqury v1.0 and Meryl v1.0 release were used in this manuscript. The versions of Merqury and Meryl used in this manuscript are available for download at each GitHub pages and at Zenodo, https://doi.org/10.5281/zenodo.3908802 [50]. Downloadable links to the Meryl k-mer databases of the assembled individuals and hap-mers are available for $A$. thaliana and NA12878 on the Merqury GitHub page [48].

\section{Ethics approval and consent to participate}

Not applicable.

\section{Consent for publication}

Not applicable.

\section{Competing interests}

S.K. has received travel compensation to present at Oxford Nanopore meetings. All other authors have no competing interests to declare.

Received: 31 March 2020 Accepted: 6 August 2020

Published online: 14 September 2020

\section{References}

1. Eid J, et al. Real-time DNA sequencing from single polymerase molecules. Science. 2009;323:133-8.

2. Wenger $\mathrm{AM}$, et al. Accurate circular consensus long-read sequencing improves variant detection and assembly of a human genome. Nat Biotechnol. 2019;37:1155-62.

3. Jain $M$, et al. Nanopore sequencing and assembly of a human genome with ultra-long reads. Nat Biotechnol. 2018;36: $338-45$.

4. Weisenfeld NI, Kumar V, Shah P, Church DM, Jaffe DB. Direct determination of diploid genome sequences. Genome Res. 2017. https://doi.org/10.1101/gr.214874.116.

5. Dekker J, Rippe K, Dekker M, Kleckner N. Capturing chromosome conformation. Science. 2002;295:1306-11.

6. Falconer $E_{\text {, et }}$ al. DNA template strand sequencing of single-cells maps genomic rearrangements at high resolution. Nat Methods. 2012;9:1107-12.

7. Pendleton $\mathrm{M}$, et al. Assembly and diploid architecture of an individual human genome via single-molecule technologies. Nat Methods. 2015;12:780-6.

8. Seo J-S, et al. De novo assembly and phasing of a Korean human genome. Nature. 2016;538:243-7.

9. Chin C-S, et al. Phased diploid genome assembly with single-molecule real-time sequencing. Nat Methods. 2016;13: 1050-4.

10. Koren S, et al. De novo assembly of haplotype-resolved genomes with trio binning. Nat Biotechnol. 2018;36:1174-82.

11. Ghareghani $M$, et al. Strand-seq enables reliable separation of long reads by chromosome via expectation maximization. Bioinformatics. 2018;34:1115-23.

12. Kronenberg ZN, et al. Extended haplotype phasing of de novo genome assemblies with FALCON-Phase. 2018. http:// biorxiv.org/lookup/doi/10.1101/327064; https://doi.org/10.1101/327064.

13. Garg, S. et al. Efficient chromosome-scale haplotype-resolved assembly of human genomes. bioRxiv 810341. 2019. https://doi.org/10.1101/810341.

14. Porubsky, D. et al. A fully phased accurate assembly of an individual human genome. 2019. http://biorxiv.org/lookup/ doi/10.1101/855049; https://doi.org/10.1101/855049.

15. Bickhart DM, et al. Single-molecule sequencing and chromatin conformation capture enable de novo reference assembly of the domestic goat genome. Nat Genet. 2017;49:643-50.

16. Simão FA, Waterhouse RM, loannidis P, Kriventseva EV, Zdobnov EM. BUSCO: assessing genome assembly and annotation completeness with single-copy orthologs. Bioinforma. Oxf. Engl. 2015;31:3210-2.

17. Mapleson D, Garcia Accinelli G, Kettleborough G, Wright J, Clavijo BJ. KAT: a K-mer analysis toolkit to quality control NGS datasets and genome assemblies. Bioinformatics. 2017;33:574-6.

18. Mikheenko A, Prjibelski A, Saveliev V, Antipov D, Gurevich A. Versatile genome assembly evaluation with QUAST-LG. Bioinforma Oxf Engl. 2018;34:1142-50.

19. Mikheenko, A., Bzikadze, A. V., Gurevich, A., Miga, K. H. \& Pevzner, P. A. TandemMapper and TandemQUAST: mapping long reads and assessing/improving assembly quality in extra-long tandem repeats. bioRxiv 2019.12.23.887158. 2019 https://doi.org/10.1101/2019.12.23.887158.

20. Choi Y, Chan AP, Kirkness E, Telenti A, Schork NJ. Comparison of phasing strategies for whole human genomes. PLoS Genet. 2018;14:e1007308.

21. Chaisson MJP, et al. Multi-platform discovery of haplotype-resolved structural variation in human genomes. Nat Commun. 2019;10:1-16.

22. Edge P, Bafna V, Bansal V. HapCUT2: robust and accurate haplotype assembly for diverse sequencing technologies. Genome Res. 2017;27:801-12.

23. Patterson $M$, et al. WhatsHap: weighted haplotype assembly for future-generation sequencing reads. J Comput Biol J Comput Mol Cell Biol. 2015;22:498-509.

24. Zook JM, et al. Integrating human sequence data sets provides a resource of benchmark SNP and indel genotype calls. Nat Biotechnol. 2014;32:246-51.

25. Zook JM, et al. Extensive sequencing of seven human genomes to characterize benchmark reference materials. Sci Data. 2016;3:1-26.

26. Krusche $P$, et al. Best practices for benchmarking germline small-variant calls in human genomes. Nat Biotechnol. 2019; 37:555-60.

27. Eberle MA, et al. A reference data set of 5.4 million phased human variants validated by genetic inheritance from sequencing a three-generation 17-member pedigree. Genome Res. 2017;27:157-64. 
28. Miga, K. H. et al. Telomere-to-telomere assembly of a complete human X chromosome. Nature 1-9. 2020. https://doi. org/10.1038/s41586-020-2547-7.

29. Dilthey A, Cox C, Iqbal Z, Nelson MR, McVean G. Improved genome inference in the MHC using a population reference graph. Nat Genet. 2015;47:682-8.

30. Sherman, R. M. \& Salzberg, S. L. Pan-genomics in the human genome era. Nat. Rev. Genet. 1-12. 2020. https://doi.org/ 10.1038/s41576-020-0210-7.

31. Human Pangenome Reference Consortium. https://humanpangenome.org/. Accessed 23 Aug 2020.

32. Rhie, A. et al. Towards complete and error-free genome assemblies of all vertebrate species. 2020. https://doi.org/10. 1101/2020.05.22.110833. Accessed 23 Aug 2020.

33. Koren, S. et al. Canu: scalable and accurate long-read assembly via adaptive k-mer weighting and repeat separation. Genome Res. gr.215087.116. 2017. https://doi.org/10.1101/gr.215087.116.

34. Chin C-S, et al. Nonhybrid, finished microbial genome assemblies from long-read SMRT sequencing data. Nat Methods. 2013;10:563-9.

35. Guan, D. et al. Identifying and removing haplotypic duplication in primary genome assemblies Bioinformatics. 2020. https://doi.org/10.1093/bioinformatics/btaa025.

36. Vurture GW, et al. GenomeScope: fast reference-free genome profiling from short reads. Bioinformatics. 2017;33:2202-4.

37. Robinson JT, et al. Integrative Genomics Viewer. Nat Biotechnol. 2011;29:24-6.

38. Thorvaldsdóttir H, Robinson JT, Mesirov JP. Integrative genomics viewer (IGV): high-performance genomics data visualization and exploration. Brief Bioinform. 2013;14:178-92.

39. Ondov BD, et al. Mash Screen: high-throughput sequence containment estimation for genome discovery. Genome Biol. 2019;20:232.

40. Quail MA, et al. A large genome center's improvements to the Illumina sequencing system. Nat Methods. 2008;5:1005-10.

41. Kozarewa I, et al. Amplification-free Illumina sequencing-library preparation facilitates improved mapping and assembly of (G+C)-biased genomes. Nat Methods. 2009;6:291-5.

42. Oyola SO, et al. Optimizing illumina next-generation sequencing library preparation for extremely at-biased genomes. BMC Genomics. 2012;13:1.

43. Miller JR, et al. Aggressive assembly of pyrosequencing reads with mates. Bioinformatics. 2008;24:2818-24.

44. Fofanov $Y$, et al. How independent are the appearances of $n$-mers in different genomes? Bioinformatics. 2004;20:2421-8.

45. Ewing B, Hillier L, Wendl MC, Green P. Base-calling of automated sequencer traces using phred. I Accuracy assessment. Genome Res. 1998:8:175-85.

46. Li, H. Aligning sequence reads, clone sequences and assembly contigs with BWA-MEM. 2013. https://arxiv.org/abs/13 03.3997v2. Accessed 23 Aug 2020.

47. Garrison, E. \& Marth, G. Haplotype-based variant detection from short-read sequencing. 2012. https://arxiv.org/abs/12 07.3907. Accessed 23 Aug 2020.

48. Rhie, A. Evaluate genome assemblies with k-mers and more. Github. 2020. https://github.com/marbl/merqury. Accessed 23 Aug 2020.

49. Walenz, B. et al. A genomic k-mer counter (and sequence utility) with nice features. Github. 2020. https://github.com/ $\mathrm{marbl} / \mathrm{meryl}$. Accessed 23 Aug 2020.

50. Rhie, A. and Walenz, B. et al. Merqury and Meryl Zenodo. 2020. https://doi.org/10.5281/zenodo.3908800.

\section{Publisher's Note}

Springer Nature remains neutral with regard to jurisdictional claims in published maps and institutional affiliations.

Ready to submit your research? Choose BMC and benefit from:
- fast, convenient online submission
- thorough peer review by experienced researchers in your field
- rapid publication on acceptance
- support for research data, including large and complex data types
- gold Open Access which fosters wider collaboration and increased citations
- maximum visibility for your research: over 100M website views per year
At BMC, research is always in progress.
Learn more biomedcentral.com/submissions

